\title{
Candida auris in Germany and Previous Exposure to Foreign Healthcare
}

\section{Axel Hamprecht, Amelia E. Barber, Sibylle C. Mellinghoff, Philipp Thelen, Grit Walther, Yanying Yu, Priya Neurgaonkar, Thomas Dandekar, Oliver A. Cornely, Ronny Martin, Oliver Kurzai, on behalf of the German Candida auris Study Group ${ }^{1}$}

Author affiliations: German Centre for Infection Research, Cologne, Germany (A. Hamprecht, S.C. Mellinghoff,

O.A. Cornely); University of Cologne, Cologne (A. Hamprecht, O.A. Cornely); Leibniz Institute for Natural Product Research and Infection Biology-Hans-Knoell-Institute, Jena, Germany (A.E. Barber, G. Walther, O. Kurzai); University Hospital Cologne, Cologne (S.C. Mellinghoff, P. Thelen); University of Würzburg, Würzburg, Germany (Y. Yu, P. Neurgaonkar, T. Dandekar, R. Martin, O. Kurzai)

DOI: https://doi.org/10.3201/eid2509.190262

The emerging yeast Candida auris has disseminated worldwide. We report on 7 cases identified in Germany during 2015-2017. In 6 of these cases, C. auris was isolated from patients previously hospitalized abroad. Whole-genome sequencing and epidemiologic analyses revealed that all patients in Germany were infected with different strains.

C Yandida auris is an emerging yeast that was initially described in 2009 after a case of otitis externa in Japan (1). Since then, healthcare-associated infections have been reported worldwide (2). C. auris has caused outbreaks in hospitals in Asia, Africa, and Latin America (2-4). In Europe, 620 C. auris cases were observed during 2013-2017 (24\% infections, $76 \%$ colonizations), including 7 cases in Germany (5). Most C. auris isolates exhibit resistance to fluconazole, and susceptibility to other azoles, amphotericin B, and echinocandins varies among isolates. Some strains show resistance to all 3 classes of antifungal drugs (6).

We report on the occurrence of $C$. auris in Germany and its link to prior healthcare exposure in the Middle East, Asia, Africa, or the United States. C. auris was isolated from 7 patients ( 4 male, 3 female, all in different, unrelated hospitals) during November 2015-December 2017 (Appendix Table, http://wwwnc.cdc.gov/EID/article/25/9/190262-App1.pdf). Six of the patients had previously been treated in healthcare centers outside Germany and were transferred to Germany for further treatment. No further suspicious cases or isolates were reported to the National

${ }^{1}$ Group members are listed at end of this article.
Reference Centre for Fungal Infections (Jena, Germany); however, reporting is not mandatory, and the possibility of missed cases cannot be excluded.

Of the 7 patients, 3 had been in isolation before detection of $C$. auris as a result of known colonization with carbapenemase-producing Enterobacteriaceae. No secondary C. auris cases were detected in any of the hospitals until March 2019. However, because no contact screening was performed, transmission resulting in asymptomatic carriage cannot be excluded.

Isolates from 6 patients were available for further testing. Biochemical identification of isolates by API ID 32C resulted in misidentification as $C$. sake (5 of 6) or C. intermedia (1 of 6). In contrast to previous versions, Vitek 2 version 08.01 (bioMérieux, https://www.biomerieux-diagnostics. com) identified all isolates as C. auris with $93 \%-99 \%$ likelihood. With VitekMS (bioMérieux) matrix-assisted laser desorption ionization time-of-flight (MALDI-TOF) mass spectrometry, no identification was achieved. However, a recent database update for VitekMS (version 3.2), which was not available at the time of our testing, corrected the identification failure in the VitekMS (data not shown). The Bruker Biotyper system (https://www.bruker.com) correctly identified all strains, albeit some with a low score (1.6-1.99). Whereas Bruker recommends that a score of 2.0 be used for species identification, a score $>1.7$ has been shown to be sufficient for reliable species identification (7). At the time of testing, Bruker's research-use-only library did not include a C. auris strain of the South Asian clade, which most of the German isolates belong to. Because C. auris exhibits considerable heterogeneity of mass spectra between geographic clusters, this missing clade likely explains the low scores (8).

Molecular identification using internal transcribed spacer technology identified all C. auris strains with $100 \%$ identity to the reference strain DSM 21092/CBS 10913. For available isolates, we performed whole-genome sequencing and aligned reads to the B8441 v2 reference genome (Figure; Appendix). A phylogenetic tree generated from whole-genome single-nucleotide polymorphism (SNP) data indicated that the isolates NRZ-2015-214, NRZ-2017-288, NRZ-2017-367, NRZ-2017-394/1-2, and NRZ-2017-505 belong to the South Asian clade, whereas NRZ-2018-545 was related to the African clade (Figure). In line with previous studies, the genetic differences observed between isolates of the same clade were small (30800 SNPs), whereas differences between clades were large (36,000-147,000 SNPs) $(4,9)$. Whole-genome data show that all cases identified in Germany harbor unique isolates, thus excluding transmission between these patients (Figure). As a control, the clonality of serial isolates NRZ2017-394/1 and NRZ-2017-394/2, taken from the same patient on 2 different occasions, was confirmed; the 2 isolates were separated by only a single SNP (Figure). 


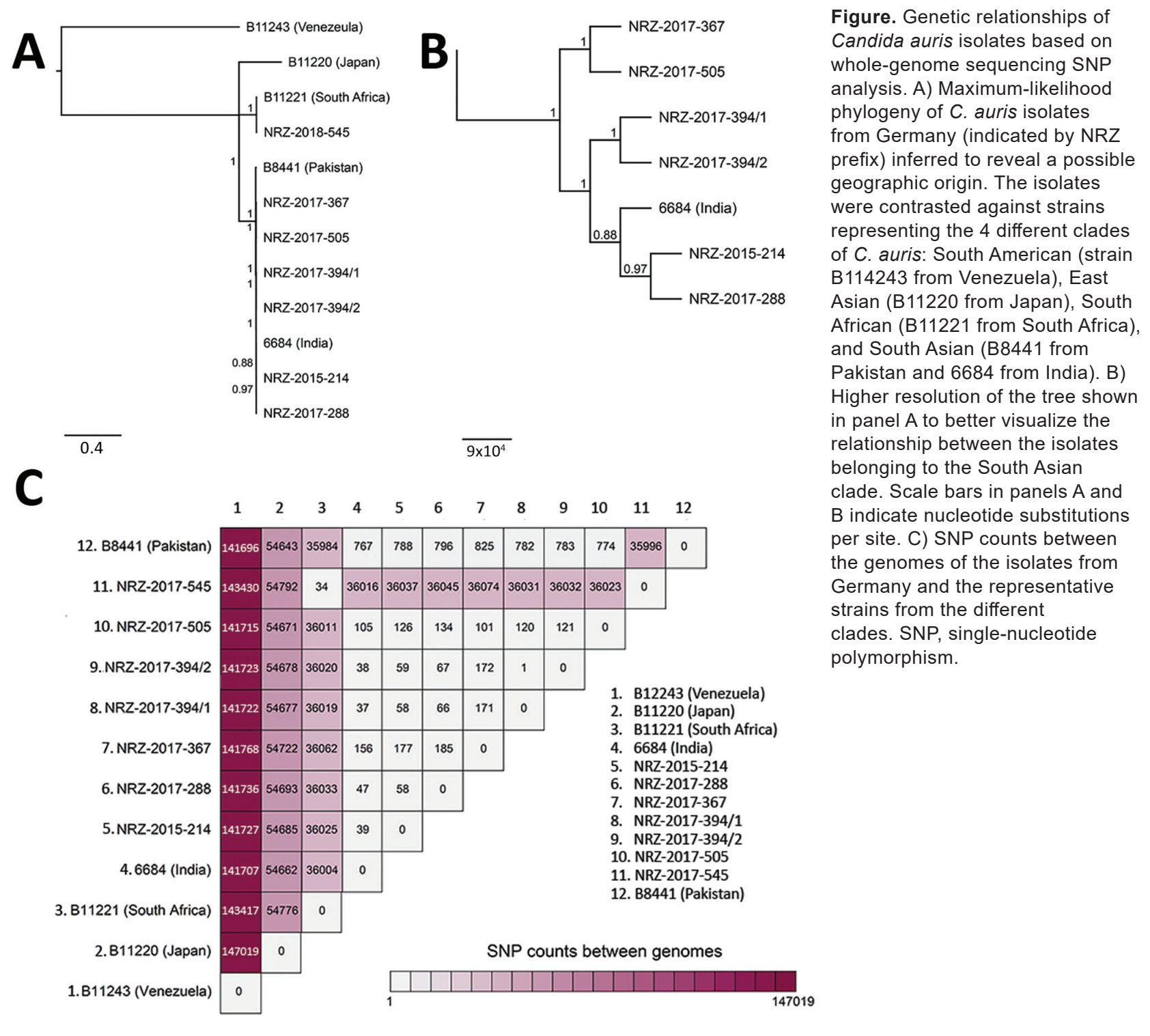

Figure. Genetic relationships of Candida auris isolates based on from Germany (indicated by NRZ prefix) inferred to reveal a possible geographic origin. The isolates of $C$. auris: South American (strain B114243 from Venezuela), East Asian (B11220 from Japan), South African (B11221 from South Africa) South Asian (B8441 from in panel $\mathrm{A}$ to better visualize the relationship between the isolates belonging to the South Asian clade. Scale bars in panels A and per site. C) SNP counts between the genomes of the isolates from Germany and the representative strains from the different clades. SNP, single-nucleotide polymorphism.

MICs of fluconazole were high for all isolates $(>64$ $\mathrm{mg} / \mathrm{L}$ ), wheareas the MICs for other antifungals were variable (Appendix Table). With the exception of NRZ-2017545 , all isolates carried either the Y132F or the K143R mutations in the ERG11 gene. However, although these mutations are linked to azole resistance, they did not result in elevated MICs for all azoles in these isolates (Appendix Table) (10). We identified a S639Y mutation in the FKS1 hotspot 1 of isolate NRZ-2017-505, which was highly resistant to anidulafungin (MIC $16 \mathrm{mg} / \mathrm{L}$ ).

In conclusion, $C$. auris has so far been isolated from individual cases in Germany. Most of these cases (6 of 7) occurred in patients who had previously been hospitalized abroad and were admitted to hospitals in Germany for continuation of medical treatment. No information on contact or environmental screening was available; such screening was likely not performed in most institutions. Thus, silent transmission and resulting carriage may have occurred unnoticed. However, no secondary cases were detected in any of the 7 hospitals affected.

Members of the German Candida auris Study Group: Gerhard Haase (Labordiagnostisches Zentrum, Universitätsklinikum RWTH Aachen, Aachen, Germany); Jette Jung (Max von Pettenkofer-Institut, Munich, Germany); Heike Freidank (Städtisches Klinikum München GmbH, Munich); Michaela Simon (Institut für Med. Mikrobiologie und Hygiene, Regensburg, Germany); Jörg Steinmann (Institut für Klinikhygiene, Medizinische Mikrobiologie und Klinische Infektiologie, Universitätsinstitut der Paracelsus Medizinischen Privatuniversität, Nuremberg, Germany). 


\section{Acknowledgments}

We thank Shneh Sethi for helpful advice and Sabrina Mündlein, Grit Mrotzek, and Ahmad Saleh for excellent technical assistance.

The German National Reference Center NRZMyk is funded by the Robert Koch Institute from funds provided by the German Ministry of Health (grant no. 1369-240). Calculations were performed on the Freiburg Galaxy server using computing services provided by the Center of Genetic Epidemiology (Danish Technical University, Lyngby, Denmark). The Freiburg Galaxy project is supported by the Collaborative Research Centre 992 Medical Epigenetics (DFG grant no. SFB 992/1 2012) and German Federal Ministry of Education and Research (BMBF grant no. 031 A538A). T.D. acknowledges support by the Deutsche Forschungsgemeinschaft (project no.

210879364-TRR 124/B1).

\section{About the Author}

Dr. Hamprecht is a clinical microbiologist at the Institute for Medical Microbiology, Immunology and Hygiene and professor for antibiotic resistance of gram-negative pathogens at the University of Cologne, Germany, and the German Centre for Infection Research (DZIF), also in Cologne. His research interests include multidrug-resistant organisms (mainly Enterobacterales and fungi), their resistance mechanisms, and the improvement of diagnostic methods.

\section{References}

1. Satoh K, Makimura K, Hasumi Y, Nishiyama Y, Uchida K, Yamaguchi H. Candida auris sp. nov., a novel ascomycetous yeast isolated from the external ear canal of an inpatient in a Japanese hospital. Microbiol Immunol. 2009;53:41-4. http://dx.doi.org/10.1111/j.1348-0421.2008.00083.x

2. Chowdhary A, Voss A, Meis JF. Multidrug-resistant Candida auris: "new kid on the block" in hospital-associated infections? J Hosp Infect. 2016;94:209-12. http://dx.doi.org/10.1016/j.jhin.2016.08.004

3. Schelenz S, Hagen F, Rhodes JL, Abdolrasouli A, Chowdhary A, Hall A, et al. First hospital outbreak of the globally emerging Candida auris in a European hospital. Antimicrob Resist Infect Control. 2016;5:35. http://dx.doi.org/10.1186/s13756-016-0132-5

4. Lockhart SR, Etienne KA, Vallabhaneni S, Farooqi J, Chowdhary A, Govender NP, et al. Simultaneous emergence of multidrugresistant Candida auris on 3 continents confirmed by wholegenome sequencing and epidemiological analyses. Clin Infect Dis. 2017;64:134-40. http://dx.doi.org/10.1093/cid/ciw691

5. Kohlenberg A, Struelens MJ, Monnet DL, Plachouras D; The Candida auris Survey Collaborative Group. Candida auris: epidemiological situation, laboratory capacity and preparedness in European Union and European Economic Area countries, 2013 to 2017. Euro Surveill. 2018;23:18-00136. http://dx.doi.org/ 10.2807/1560-7917.ES.2018.23.13.18-00136

6. Jeffery-Smith A, Taori SK, Schelenz S, Jeffery K, Johnson EM, Borman A, et al.; Candida auris Incident Management Team. Candida auris: a review of the literature. Clin Microbiol Rev. 2017;31:e00029-17. http://dx.doi.org/10.1128/CMR.00029-17

7. Hamprecht A, Christ S, Oestreicher T, Plum G, Kempf VA, Göttig S. Performance of two MALDI-TOF MS systems for the identification of yeasts isolated from bloodstream infections and cerebrospinal fluids using a time-saving direct transfer protocol.
Med Microbiol Immunol (Berl). 2014;203:93-9. http://dx.doi.org/ 10.1007/s00430-013-0319-9

8. Prakash A, Sharma C, Singh A, Kumar Singh P, Kumar A, Hagen F, et al. Evidence of genotypic diversity among Candida auris isolates by multilocus sequence typing, matrix-assisted laser desorption ionization time-of-flight mass spectrometry and amplified fragment length polymorphism. Clin Microbiol Infect. 2016;22:277e1-9. http://dx.doi.org/10.1016/j.cmi.2015.10.022

9. Magobo RE, Corcoran C, Seetharam S, Govender NP. Candida auris-associated candidemia, South Africa. Emerg Infect Dis. 2014;20:1250-1. http://dx.doi.org/10.3201/eid2007.131765

10. Chowdhary A, Prakash A, Sharma C, Kordalewska M, Kumar A, Sarma S, et al. A multicentre study of antifungal susceptibility patterns among 350 Candida auris isolates (2009-17) in India: role of the ERG11 and FKS1 genes in azole and echinocandin resistance. J Antimicrob Chemother. 2018;73:891-9. http://dx.doi.org/10.1093/jac/dkx480

Address for correspondence: Oliver Kurzai, University of Würzburg Institute for Hygiene and Microbiology, Josef-Schneider-Straße 2 / E1, Würzburg 97080, Germany; email: okurzai@hygiene.uni-wuerzburg.de

\section{Characterization of Clinical Isolates of Talaromyces marneffei and Related Species, California, USA}

\section{Linlin Li, Katelyn Chen, Nirmala Dhungana, Yvonne Jang, Vishnu Chaturvedi, ${ }^{1}$ Ed Desmond ${ }^{2}$}

Author affiliation: California Department of Public Health, Richmond, California, USA

DOI: https://doi.org/10.3201/eid2509.190380

Talaromyces marneffei and other Talaromyces species can cause opportunistic invasive fungal infections. We characterized clinical Talaromyces isolates from patients in California, USA, a non-Talaromyces-endemic area, by a multiphasic approach, including multigene phylogeny, matrix-assisted laser desorption/ionization time-of-flight mass spectrometry, and phenotypic methods. We identified 10 potentially pathogenic Talaromyces isolates, 2 T. marneffei.

\footnotetext{
${ }^{1}$ Current affiliation: New York State Department of Health, Albany, New York, USA.

${ }^{2}$ Current affiliation: Hawaii State Department of Health, Pearl City, Hawaii, USA.
} 